\title{
The primacy of multiparametric MRI in men with suspected prostate cancer
}

\author{
Jonathan Richenberg $^{1} \cdot$ Vibeke Løgager $^{2} \cdot$ Valeria Panebianco $^{3} \cdot$ Olivier Rouviere $^{4,5} \cdot$ Geert Villeirs $^{6} \cdot$ Ivo G. Schoots $^{7}$
}

Received: 15 November 2018 / Revised: 7 March 2019 / Accepted: 14 March 2019 / Published online: 6 June 2019

(C) The Author(s) 2019

\begin{abstract}
Background Multiparametric MRI (mpMRI) became recognised in investigating those with suspected prostate cancer between 2010 and 2012; in the USA, the preventative task force moratorium on PSA screening was a strong catalyst. In a few short years, it has been adopted into daily urological and oncological practice. The pace of clinical uptake, born along by countless papers proclaiming high accuracy in detecting clinically significant prostate cancer, has sparked much debate about the timing of mpMRI within the traditional biopsy-driven clinical pathways. There are strongly held opposing views on using mpMRI as a triage test regarding the need for biopsy and/or guiding the biopsy pattern.

Objective To review the evidence base and present a position paper on the role of mpMRI in the diagnosis and management of prostate cancer.

Methods A subgroup of experts from the ESUR Prostate MRI Working Group conducted literature review and face to face and electronic exchanges to draw up a position statement.

Results This paper considers diagnostic strategies for clinically significant prostate cancer; current national and international guidance; the impact of pre-biopsy mpMRI in detection of clinically significant and clinically insignificant neoplasms; the impact of pre-biopsy mpMRI on biopsy strategies and targeting; the notion of mpMRI within a wider risk evaluation on a patient by patient basis; the problems that beset mpMRI including inter-observer variability.

Conclusions The paper concludes with a set of suggestions for using mpMRI to influence who to biopsy and who not to biopsy at diagnosis.

\section{Key Points}

- Adopt mpMRI as the first, and primary, investigation in the workup of men with suspected prostate cancer.

- PI-RADS assessment categories 1 and 2 have a high negative predictive value in excluding significant disease, and systematic biopsy may be postponed, especially in men with low-risk of disease following additional risk stratification.

- PI-RADS assessment category lesions 4 and 5 should be targeted; PI-RADS assessment category lesion 3 may be biopsied as a target, as part of systematic biopsies or may be observed depending on risk stratification.
\end{abstract}

Keywords Prostate cancer $\cdot$ Magnetic resonance imaging $\cdot$ Biopsy $\cdot$ Risk assessment $\cdot$ Observer variation

Electronic supplementary material The online version of this article (https://doi.org/10.1007/s00330-019-06166-z) contains supplementary material, which is available to authorized users.

Jonathan Richenberg

jonathan.richenberg@bsuh.nhs.uk

1 Department of Imaging, Brighton \& Sussex University Hospitals NHS Trust and Brighton and Sussex Medical School, Brighton BN2 5BE, UK

2 Department of Radiology, Herlev University Hospital Copenhagen University, Herlev, Denmark

3 Department of Radiological Sciences, Oncology and Pathology, Sapienza, University of Rome, Rome, Italy
4 Hospices civils de Lyon, Department of Urinary and Vascular Radiology, hôpital Édouard-Herriot, 69437 Lyon, France

5 Faculté de médecine Lyon Est, Université Lyon 1, 69003 Lyon, France

6 Department of Radiology, Ghent University Hospital, Ghent, Belgium

7 Department of Radiology \& Nuclear Medicine, Erasmus MC University Medical Center Rotterdam, Rotterdam, The Netherlands 


$\begin{array}{ll}\text { Abbreviations } & \\ \text { ADC } & \text { Apparent diffusion coefficient } \\ \text { AUC } & \text { Area under the ROC curve } \\ \text { CI } & \text { Confidence interval } \\ \text { cisPCa } & \text { Clinically insignificant prostate cancer } \\ \text { csPCa } & \text { Clinically significant prostate cancer } \\ \text { DCE } & \text { Dynamic contrast-enhanced } \\ \text { DRE } & \text { Digital rectal examination } \\ \text { DWI } & \text { Diffusion-weighted imaging } \\ \text { EAU } & \text { European Association of Urology } \\ \text { EPE } & \text { Extraprostatic extension } \\ \text { ERSPC } & \text { European Randomised Study of Screening } \\ & \text { for PCa } \\ \text { ESO } & \text { European Society of Oncology } \\ \text { ESUR } & \text { European Society of Urogenital Radiology } \\ \text { GG } & \text { Grade grouping } \\ \text { GS } & \text { Gleason score } \\ \text { IQR } & \text { Interquartile range } \\ \text { mpMRI } & \text { Multiparametric magnetic resonance imaging } \\ \text { MRSI } & \text { Magnetic resonance spectroscopic imaging } \\ \text { NPV } & \text { Negative predictive value } \\ \text { PI-RADS } & \text { Prostate Imaging Reporting and Data System } \\ \text { PI-RADS v1 } & \text { Prostate Imaging Reporting and Data } \\ & \text { System version 1 } \\ \text { PI-RADS v2 } & \text { Prostate Imaging Reporting and Data } \\ & \text { System version 2 } \\ \text { PPV } & \text { Positive predictive value } \\ \text { PSA } & \text { Prostate-specific antigen } \\ \text { PSAd } & \text { Prostate-specific antigen density } \\ \text { SBx } & \text { Systematic biopsies } \\ \text { T2W } & \text { T2 weighted } \\ \text { TBx } & \text { MRI-targeted biopsies } \\ \text { TBx } & \text { Targeted biopsy } \\ \text { TRUS } & \text { Transrectal ultrasound } \\ \text { TTP Bx } & \text { Template transperineal biopsy } \\ \text { Trial } & \text { PRIAS } \\ \text { Trial } & \text { PROTECT } \\ \text { Trial } & \text { PRECISION } \\ \text { Trial } & \text { PROMIS } \\ & \end{array}$

\section{Introduction}

In 2012, the European Society of Urogenital Radiology (ESUR) prostate committee promoted the use of multiparametric MRI (mpMRI) in the routine management of men with suspected or confirmed prostate cancer [1]. That proposal has gained widespread acceptance. The debate has now moved to when mpMRI should be used.

Expressions of interest were sought from the 58 members of the ESUR Prostate MRI Working Group at the European Congress of Radiology (ECR) in March 2017 in contributing to a position statement on the use of mpMRI in prostate cancer diagnosis. Each of the 7 initial positive respondents was invited to contribute but based on the relative contributions, the final author list was revised to 5 ensuring due representation of the group's European composition; a sixth contributor joined at ECR, March 2018. The final contributors were from the UK, France, The Netherlands, Denmark, Italy, and Belgium.

The approach was to review published evidence, supplemented by knowledge of completed cohort studies in the process of being published (having been accepted for publication). In this way, there was very little intra-author disagreement. On the specific topic of biopsy planning - the pros and cons of systematic versus targeted-driven approach - there was some variation in how strongly the argument for targeted biopsy over systematic biopsy could be worded. As the paper neared completion, the results from on-going studies became known to the author group, such that a consensus position was reached.

\section{Evaluating clinically significant prostate cancer}

\section{Evaluating clinically significant prostate cancer in the pre-MRI era}

Urologists and oncologists gauge prostate cancer aggressiveness by combining DRE findings, serum PSA levels and data derived from systematic biopsy findings.

Men with suspected prostate cancer are categorised into risk groups (see EAU risk classification in EAU guidelines on prostate cancer [2]). This classification is based on the grouping of patients with a similar risk of biochemical recurrence after radical treatment [3]. Tables and nomograms have been developed to predict the likelihood of extraprostatic spread, seminal vesicle invasion and lymph node involvement, and some even state recurrence-free survival rates at 3 and 5 years [4-8].

There is, however, still no consensus of what constitutes a clinically significant prostate cancer (csPCa) [9]. Current argument centres on Gleason category 7 pattern 3 or 4 dominance, ISUP grades 2 and 3, respectively. While the 2014 grading system differentiates Gleason 7 by dominant pattern, all Gleason 7 is classed intermediate risk, albeit with the qualification that emerging clinical data support the distinction between favourable (ISUP grade 2) and unfavourable-risk (ISUP grade 3) patient categories within the intermediate-risk group $[2,10,11]$.

\section{Evaluating csPCa in the post-MRI era}

mpMRI can detect and localise cancers with a Gleason score $\geq 7$ more easily than lower-grade cancers [12-15], relying on the lower signal intensity of higher-grade cancers on T2weighted imaging (T2w), more impeded diffusion on diffusion-weighted imaging (DWI), early enhancement on dynamic contrast sequences (DCE), and (previously) higher 
choline over citrate ratios on spectroscopic imaging [16-18]. mpMRI evaluates lesion volume with reasonable accuracy, at least for aggressive tumours [19]. In one study, correlations between lesion volume estimated on T2-weighted images, ADC maps, and DCE-MR images with pathology were 0.91 and 0.93 , respectively [20].

In 2012, the ESUR proposed a standardised reporting tool called 'PI-RADS' (Prostate Imaging Reporting and Data System) [1] in an attempt to align mpMRI findings with the risk of having csPCa. In 2015, an updated version (PI-RADS v2) was published in collaboration with the American College of Radiology and the AdMeTech Foundation [21, 22] (Table 1). PI-RADS 2 has been validated in a meta-analysis of 21 studies including over 3857 patients. This demonstrated a pooled sensitivity of $89 \%$ and a pooled specificity of $73 \%$ [23].

The EAU/ESTRO/ESUR/SIOG recommends using mpMRI before repeat biopsy, combining a TRUS-directed diagnostic approach with the addition of the mpMRI and subsequently targeted biopsies [2, 24]. Neither the European (EAU/ ESTRO/SIOG/ESUR) nor the American (NCCN) guidelines endorse wholeheartedly mpMRI in biopsy-naïve men [2, 24].

The NICE guideline CG175 [25] has been updated and is due for publication in April 2019 [https://www.nice.org.uk/ guidance/indevelopment/gid-ng10057]; it recommends prebiopsy mpMRI, putting mpMRI as the primary method to investigate those with suspected prostate cancer based on PSA and/or DRE findings. Revised in November 2018, the French guidelines now also recommend pre-biopsy mpMRI for all, including biopsy-naive [26]. Appendix 1, which includes in addition to references cited in the main text citations to PROTECT trial [27], Belgian National Guidance [28] and recently updated French National Guidance [29].

Eligibility criteria to have an mpMRI (in place of biopsy) should be based on the EAU and/or National current recommendations for biopsy referrals. Following suitable clinical evaluation for acute or chronic reasons not to be investigated, the reasons to offer mpMRI would mirror those currently used to offer biopsy.

\section{Pre-biopsy mpMRI advantages}

The problem with any approach dominated by TRUS-guided systematic biopsy ( $\mathrm{SBx}$ ) is that it is organ rather than lesion based, introducing two major limitations: overdiagnosis of clinically insignificant prostate cancers (cisPCa) and underdiagnosis of csPCa.

\section{The case for excluding men from biopsy based on mpMRI}

Avoiding or deferring biopsy (possibly indefinitely) if mpMRI suggests low likelihood of csPCa would reduce the burden to men and to their health systems of initial diagnostic workup and low-grade prostate cancer follow-up. Such an approach may also improve the cost efficiency of the diagnostic workup [30]. Accepting that the results are subject to assumptions around test costs, sensitivity of mpMRI-influenced biopsies, and long-term outcomes of men with $\mathrm{PCa}$, recent analysis of a UK population concluded mpMRI first followed by up to 2 rounds of biopsy is more cost effective than current practice [31]. This strategy requires a high negative predictive value (NPV) of mpMRI in excluding csPCa.

A recent systematic review (9613 men) in conjunction with the EAU-ESTRO-ESUR Prostate Cancer Guidelines panel revealed a median mpMRI NPV of $82 \%$ (interquartile range (IQR), 69-92\%) for overall cancer exclusion and of $88 \%$ (IQR, 86-92\%) for csPCa exclusion [32]. The critical issue highlighted in this review is that the reported range of the NPV for mpMRI is extreme and varies according to definitions and risk categorisation used.

A key variable of the NPV is the prevalence of cancer within the population being monitored: when the prevalence doubles from 30 to $60 \%$, the NPV of mpMRI (scores 1-2 taken as 'negative') falls from 88 to $67 \%$ (for any cancer grade) (Table 2) [32]. NPV therefore is bound to be variable as it depends on whether mpMRI is being used in a low-risk screening setting or in a

Table 1 Comparison of Prostate Imaging and Reporting and Data System versions 1 and 2 (adapted from Barentsz et al [22])

\begin{tabular}{ll}
\hline PI-RADS version 1 & PI-RADS version 2 \\
\hline $\begin{array}{l}\text { A sum score of 3-15 (20 with MRSI) for T2W + DWI + DCE } \\
\text { (+ MRSI) is suggested }\end{array}$ & $1-5$ point dominant score \\
& For peripheral zone, DWI is dominant \\
& For transition zone, T2W is dominant \\
Equal role for DCE (5-point scale) & Secondary role for DCE (positive or negative) \\
For DWI: ADC images are mandatory & For DWI: ADC and high $b$ value images $(b$ value $>1400)$ are mandatory \\
27-sector map & 39-sector map \\
MRSI can be included & MRSI is not included \\
Size is not used for T2W + DWI & Size $(>15$ mm) is used for T2W + DWI to separate PI-RADS scores 4 and 5 \\
\hline
\end{tabular}


Table 2 Negative predictive estimates for pre-biopsy mpMRI as a function of prostate cancer prevalence (adapted from Molovan et al [32])

\begin{tabular}{ll}
\hline PCa prevalence & NPV \\
\hline 0.30 & $0.88(0.77-0.99)$ \\
0.40 & $0.82(0.70-0.94)$ \\
0.50 & $0.76(0.64-0.88)$ \\
0.60 & $0.67(0.56-0.79)$ \\
0.70 & $0.57(0.47-0.67)$ \\
\hline
\end{tabular}

selected high-risk cohort. Furthermore, the prevalence will alter according to the definition of csPCa (Table 3).

Three pivotal multicentre trials on the use of mpMRI in biopsy-naïve men inform this review: PROMIS, PRECISION [33, 34], and the 4M study by van der Leest [35].

The PROMIS trial assessed mpMRI, 12-core SBx and template transperineal biopsy (TTP Bx) in 576 prospectively included biopsy-naïve men [33]. Forty percent of patients had csPCa (defined as Gleason score $\geq 4+3$ or cancer core length $\geq 6 \mathrm{~mm}$ ) at TTP (Table 3). Using TTP Bx as a reference test, the NPV for detecting csPCa was 0.89 (95\% CI, 0.83-0.94) for mpMRI compared with $0.74(0.69-0.78)$ for TRUS SBx (csPCa prevalence,: $40 \%$ (95\% CI, 36-44\%)). mpMRI failed to report 7\% (17/230) Gleason $3+4$ cancers with core lengths between 6 and $12 \mathrm{~mm}$, but no Gleason $4+3$ or worse cancers. When accepting missing this $7 \%$, mpMRI (used as a triage test) could have avoided $27 \%$ of primary biopsies, while detecting $18 \%$ more csPCa and 'missing' $5 \%$ of cisPCa [33]. The definition of csPCa propagated by the START consortium is GS $\geq 3+4$ [36]. The usage of this definition in the PROMIS study showed an increase in the prevalence of csPCa to $53 \%$ (49-58\%); the NPV dropped to $76 \%$ (69-82\%). mpMRI failed to report $12 \%$ Gleason $3+4$ prostate cancers, but still could have avoided $27 \%$ of primary biopsies.

Similar results were reported in the more recent multicentre, randomised, noninferiority PRECISION trial, in which 500 biopsy-naïve men were randomised to undergo either mpMRI with or without targeted biopsy, or standard transrectal ultrasonography-guided biopsy [34]. Using mpMRI as a triage test could have avoided $28 \%$ of primary biopsies, while detecting $12 \%$ more csPCa (defined as Gleason score $\geq 3+4$ ) than SBx and 'missing' $13 \%$ of cisPCa. These results were obtained in 25 centres (academic and non-academic) with mixed experience in both mpMRI and MR-targeted biopsy, and without restrictions on the use of a $1.5-\mathrm{T}$ or a $3.0-\mathrm{T}$ system, endorectal coil, or biopsy technique (visual registration, software-assisted registration or in-bore).

The 4M study included 626 biopsy-naïve patients; all patients underwent systematic biopsy, and those with a positive mpMRI (PI-RADS 3-5, 51\%) underwent additional in-bore MRI-TBx. SBx performed in PI-RADS 1-2 cases detected csPCa in only $3 \%$ of the patients while detecting cisPCa in $20 \%$, with an $89 \%$ reduction in total biopsy cores [35].

In a clinical follow-up study (median follow-up of 41 months) of a mixed population of biopsy-naïve, repeat biopsies, and active surveillance $(n=300)$, who had undergone a negative in-bore MR-guided biopsy for PI-RADS 35 lesions, only $1.7 \%(5 / 300)$ had csPCa subsequently diagnosed by any kind of follow-up histology in 82 men (any biopsy or radical prostatectomy), and in 218 without any histology confirmation [37]. In another cohort of 1255 patients with negative mpMRI, the csPCa-free survival rates at 48 months were $95 \%$ in originally biopsy-naïve patients and $96 \%$ in patients with a prior negative biopsy [38].

\section{Improving detection of csPCa}

Pre-biopsy mpMRI in men with suspected prostate cancer is justified further if it improves the detection of csPCa through targeted biopsies of any suspicious lesion suggested by mpMRI.

Radiologic-pathologic correlations with whole-mounts have shown that mpMRI is highly sensitive for locating aggressive cancers, with $80-86 \%$ of Gleason 7 and $93-100 \%$ of Gleason $\geq 8$ detected [12]. Correlation studies of mpMRI with TBx or radical prostatectomy specimens performed after the introduction of PI-RADS showed that the location of the index lesion was correctly assessed by mpMRI in $95 \%$ of patients [39] and that mpMRI missed $10 \%$ csPCa on a per-lesion basis [40]. mpMRI results, whether expressed as subjective (Likert) scoring [41-43], PI-RADS v1 [44, 45], or PI-RADS v2 scoring [46], were found significant predictors of the presence of csPCa at biopsy.

A recent systematic review of mostly retrospective studies showed that TBx performed under MR/TRUS fusion detected more csPCa than SBx, with a median detection rate of $33.3 \%$ (range, $13.2-50 \%$ ) versus $23.6 \%$ (range, $4.8-52 \%$ ), respectively. The absolute difference in the detection rates between the two approaches was a median of $6.8 \%$ (range, $0.9-41.4 \%$ ) and

Table 3 Diagnostic accuracy results from mpMRI for different definitions of clinically significant prostate cancer (adapted from PROMIS study [33])

\begin{tabular}{|c|c|c|c|c|c|}
\hline Definition of csPCa & Prevalence $(\%)$ & Sensitivity & Specificity & PPV & NPV \\
\hline Gleason score $\geq 3+4$ or cancer core length $\geq 4 \mathrm{~mm}$, & $57(53-62)$ & $87(83-90)$ & $47(40-53)$ & $69(64-73)$ & $72(65-79)$ \\
\hline Gleason score $\geq 3+4$ & $53(49-58)$ & $88(84-91)$ & $45(39-51)$ & $65(60-69)$ & $76(69-82)$ \\
\hline Gleason score $\geq 4+3$ or cancer core length $\geq 6 \mathrm{~mm}$ & $40(36-44)$ & $93(88-96)$ & $41(36-46)$ & $51(46-56)$ & $89(83-94)$ \\
\hline
\end{tabular}


always in favour of TBx. The median number of biopsy cores to detect one man with csPCa was 37.1 (IQR, 32.6-82.8) and 9.2 (IQR, 4-37.7) for SBx and TBx, respectively [47]. Another systematic review (focussing on MRI positive men only), including studies that used MR/TRUS fusion, cognitive guidance, or inbore guidance for TBx, also found that $\mathrm{TBx}$ has a higher rate of detection of csPCa than SBx with a sensitivity of 0.91 (95\%CI, $0.87-0.94)$ and 0.76 (95\%CI, 0.64-0.84), respectively, in a mixed population of biopsy-naïve men and men with previous negative biopsies [48]. The sensitivity (detection) ratio was 1.10 (95\%CI, 1.00-1.22) and significantly in favour of TBx for biopsy-naïve men only, and 1.54 (95\%CI, 1.05, 2.26) in men with previous negative biopsies. In a head-to-head comparison in 223 men with elevated PSA and/or abnormal DRE, mpMRIinfluenced biopsy outperformed systematic 12-core TRUS biopsy in detecting csPCa on a patient basis (42\% vs $35 \%$ ) and on a lesion basis (74\% vs 61\%) with a 'miss rate for significant lesions' of $\sim 18 \%$ in the MRI biopsy group versus $\sim 26 \%$ rate in the TRUS biopsy group [49].

\section{Targeted versus systematic biopsy versus combined approach for clinically significant prostate cancer detection}

mpMRI-targeted biopsies detect about $90 \%$ of all csPCa [12, $33,48]$. This, however, also means that about $10 \%$ of csPCa are missed if only a targeted approach is adopted. Indeed, histological correlation highlights that Gleason $\geq 7$ cancers can be invisible on mpMRI [12]. It therefore may seem prudent, on first thoughts, to supplement targeted biopsies with systematic biopsies to 'capture' any csPCa that is missed by mpMRI (usually low grade 4 and organ-confined [50], located in the dorsolateral or apical segments of the peripheral zone [49]).

The combination of SBx and MRI-targeted biopsies (TBx) comes at a cost: over-detection of cisPCa [51, 52]. In a systematic review of 16 studies comparing TBx and $\mathrm{SBx}$ in mixed populations of biopsy-naïve men and men with previous negative biopsies, this overdiagnosis was almost halved by omitting $\mathrm{SBx}$ [48]. In the PRECISION trial, $13 \%$ (95\% CI, -19 to $7 \% ; p<0.001)$ fewer men were diagnosed with cisPCa in the MRI-targeted biopsy group (in total 9\%) than in the standard biopsy group (in total 22\%); again, this diagnosis was more than halved by omitting SBx. Likewise, in a prospective nonrandomised trial of 1003 men who underwent both TBx and SBx, adding SBx to TBx identified an additional 103 (22\%) prostate cancers, $83 \%$ of which were low-grade [53].

Excluding SBx in the MRI-negative men in the 4M would have avoided biopsies in $49 \%$ in this study population at a small expense of missing csPCa [35].

The MRI-FIRST multicentre study [54] recruited biopsynaïve men $(n=251)$ under 75 years with a PSA $\leq 20 \mathrm{ng} / \mathrm{ml}$. All patients had 12-core SBx plus 2 optional cores to hypoechoic lesions by one operator blinded to mpMRI results, and TBx (up to 2 targeted lesions, 3 cores per lesion) by another operator. SBx and TBx detection rates for ISUP grade $\geq 2$ tumours were $29.9 \%$ and $32.3 \%$, respectively $(p=0.38$; detection ratio 1.08). ISUP grade $\geq 2$ cancers would have been missed in $7.6 \%(95 \% \mathrm{CI}, 4.6-11.6 \%)$ of patients if TBx had not been taken, and in $5.2 \%(95 \% \mathrm{CI}, 2.8-8.7 \%)$ of patients if SBx had not been performed. TBx detected significantly more ISUP grade $\geq 3$ tumours than $\mathrm{SBx}(19.9 \%$ vs $15.1 \%, p=$ 0.0095 ; detection ratio, 1.32 ). ISUP grade $\geq 3$ cancers would have been missed in $6.0 \%(95 \% \mathrm{CI}, 3.4-9.7 \%)$ of patients if TBx had not been taken, but in only $1.2 \%$ (95\%CI, $0.2-3.5 \%)$ of patients if SBx had not been performed. These data indicate that predominantly ISUP grade 2 prostate cancers were detected by the inclusion of SBx.

Technology that allows mapping exactly the biopsy needle path has suggested that the median number of systematic cores sampling the same region selected for target is 2 . If these 'isometric' systematic cores are disregarded, systematic biopsy has a modest benefit only: $3 \%$ cancer detection instead of benign diagnosis and $\sim 1 \%$ from cisPCa to csPCa [55].

There are few data comparing TBx to TTP Bx. TTP Bx should be considered in patients at high risk with negative mpMRI and in some patients at low-risk with persistently elevated PSA and a negative MRI.

\section{Biopsy strategy in the 'mpMRI first' era}

It is impossible to brush aside that the range of published mpMRI NPV figures is broad and that csPCa prevalence (i.e. pre-MRI probability of csPCa) has a major impact on NPV [9, 32]. Therefore, there is a need to refine the biopsy planning process by incorporating the mpMRI findings within a larger nomogram containing clinical data to determine an individual's likelihood of having csPCa.

In a recent multivariate logistic regression analysis to predict likelihood of csPCa for biopsy-naive and previously biopsied men, the PI-RADS classification contributed significantly to a newly developed risk model $(p<0.001)$ in combination with the ERSPC-RCs (www.prostatecancerriskcalculator.com) based on the European Randomised Study of Screening for PCa (ERSPC) [56, 57]. For biopsynaive men, the risk model reached a higher AUC (0.83), compared with ERSPC-RC3 (0.81), refitted RC3 (0.80), and PIRADSv1.0 (0.76). The risk model AUC was comparable with that of ERSPC-RC3 + PI-RADSv1.0 (0.84). Likelihood ratio test results similarly showed that the risk-models may perform significantly better compared with (refitted) ERSPCs and PIRADS alone. Others have confirmed these results [58].

\section{PI-RADS 3 lesions and risk stratification and biopsy}

PI-RADS assessment category 3 is assigned when the probability of prostate cancer is uncertain. The percentage of 
patients assigned a PI-RADS assessment category of 3 is extremely variable in the different published cohorts [59]. As expected, the biopsy positivity rate is also highly variable in these lesions; the cumulative total of high-grade PCa (GS $\geq$ $3+4)$ in the PI-RADS category 3 has been reported $21 \%$ (range 4-27\%) in biopsy-naïve men and 16\% (range 10$19 \%$ ) men with previous negative biopsies [59]. Another paper lists overall cancer detection $16-67 \%$ and proportion of Gleason $\geq 7$ cancers $0-43 \%$ [46]. Based on these data, the authors concluded that the prevalence of PI-RADS 3 index lesions in the diagnostic workup is not negligible, varying between one in five $(22 \%)$ and one in three (32\%) men, depending on patient cohort of the first biopsies or previous negative biopsies. The actual prevalence of csPCa after TBx in PI-RADS category 3 lesions varies between patient groups from one in five $(21 \%)$ and one in six $(16 \%)$, depending on previous biopsy status. Although this prevalence is lower in comparison to PI-RADS category 4 and PI-RADS category 5 lesions, still a considerable proportion of men harbour significant disease.

\section{Biopsy strategy and patient risk}

Biopsy decisions should first be based on mpMRI findings, favouring avoiding biopsy in 'negative' (any/all lesions PIRADS 2 or less) studies and targeting PI-RADS 4 or 5 lesions. For some 'negative' studies and most PI-RADS 3 lesions, a second assessment incorporating clinical (age, DRE, family history for example) and biochemical (PSA density and velocity) parameters should be applied to see if systematic biopsy alone or in addition to targets to the low-grade PI-RADS lesions are indicated.

The use of PSA density may improve the patient selection for biopsy [32, 35, 56, 57, 60-65]. Two studies using either the PI-RADS V1 scoring [66] or a mix of PI-RADS v1 and v2 systems [37] suggested that PSA density could discriminate among PI-RADS 3 patients, those who need to undergo prostate biopsy from those who can be followed up. In a multicentre study of biopsy-naïve men, PI-RADS category 3 lesions were further categorised into PSA density of $<0.10$, $0.10-0.20$, and $>0.20$ : the detection of GS $\geq 3+4$ PCa was $18 \%, 31 \%, 46 \%$, respectively [67].

In patients stratified into a priori low-to-intermediate risk (Table 1), the mpMRI NPV is probably sufficiently high to avoid SBx in case of negative mpMRI [34]. Systematic biopsy results would be expected to be less influential on patient management in the setting of a positive mpMRI with a suspicious lesion than in a negative mpMRI. In high-risk patients, however, patients with negative mpMRI will probably still need SBx [34]; even in expert centres, mpMRI may 'miss' $10-12 \%$ csPCa. The recommended shift towards an MRIdirected and risk-stratified approach to seeking csPCa is captured in the flowchart (Fig. 1). A shift in emphasis away from SBx with additional MRI-TBx to MRI-TBx for highprobability mpMRI examinations is recommended, accepting the modest price of missing csPCa [33, 68-75].

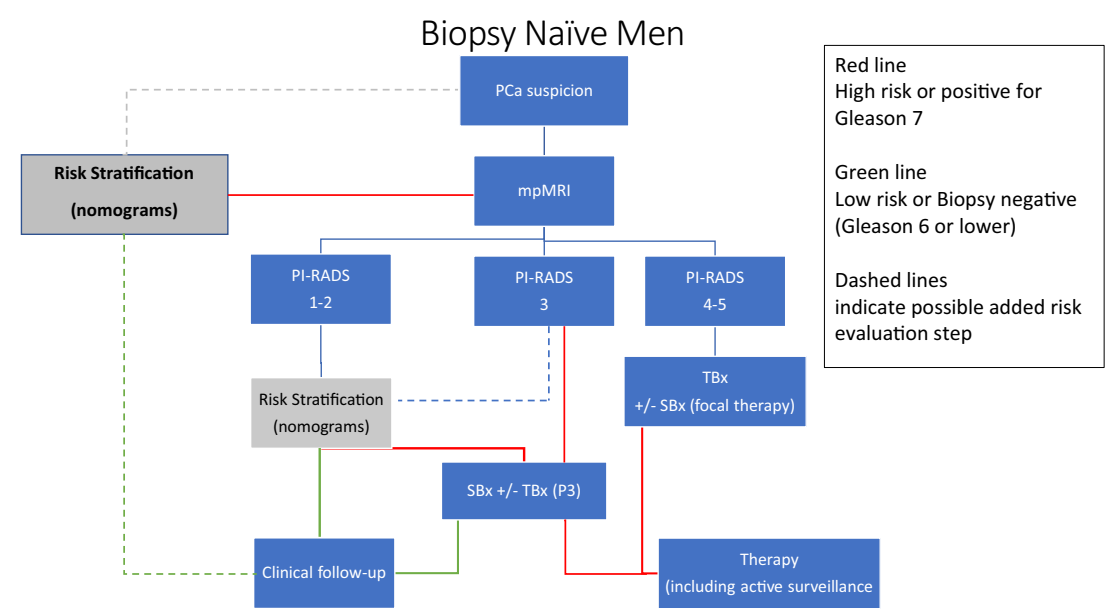

Fig. 1 Proposed flowchart for investigating men suspected having prostate cancer, beginning with mpMRI. Using mpMRI as the primary investigation in prostate cancer diagnostic workup following clinical suspicion, men will be stratified into PI-RADS assessment categories $1-2,3$, and 4-5. Capitalising on the high negative predictive value of mpMRI, assessment category 1-2 may indicate clinical follow-up avoiding systematic biopsy, or indicate further risk stratification with developing risk calculators (nomograms). Assessment category 3 may indicate MR-targeted biopsy (TBx) combined with systematic biopsy $(\mathrm{SBx})$ to gain maximal diagnostic yield. Alternatively, risk stratification may sub-differentiate these men into high-risk and low-risk; the low-risk group may defer systematic biopsy. Assessment category 4-5 may indicate MR-targeted biopsy. Systematic biopsy could be performed in direct combination or secondary, depending on biopsy workflow. In assessment category 5 , the added value of systematic biopsy would be limited. When prostate cancer has not been identified, additional risk stratification could be performed to indicate or avoid additional systematic and targeted biopsy. Green arrows, low-risk; red arrows, intermediate-/high-risk. Dotted lines indicate research in progress. PCa, prostate cancer; MRI, magnetic resonance; PI-RADS, prostate imaging reporting and data system = suspicion MRI score (1-5); TBx, MRI-targeted biopsy; $\mathrm{SBx}$, transrectal/transperineal ultrasound-guided systematic biopsy; AS, active surveillance 


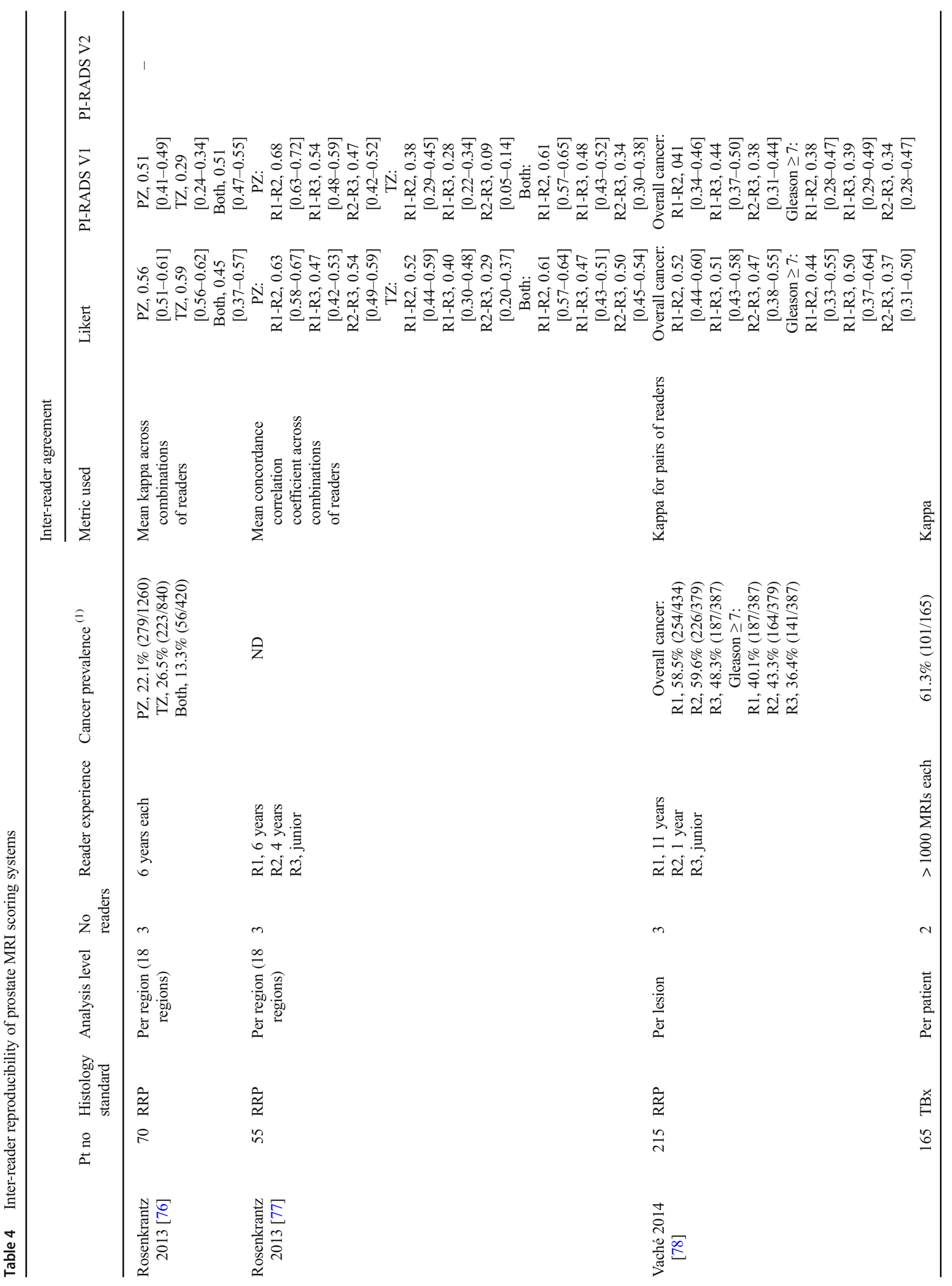




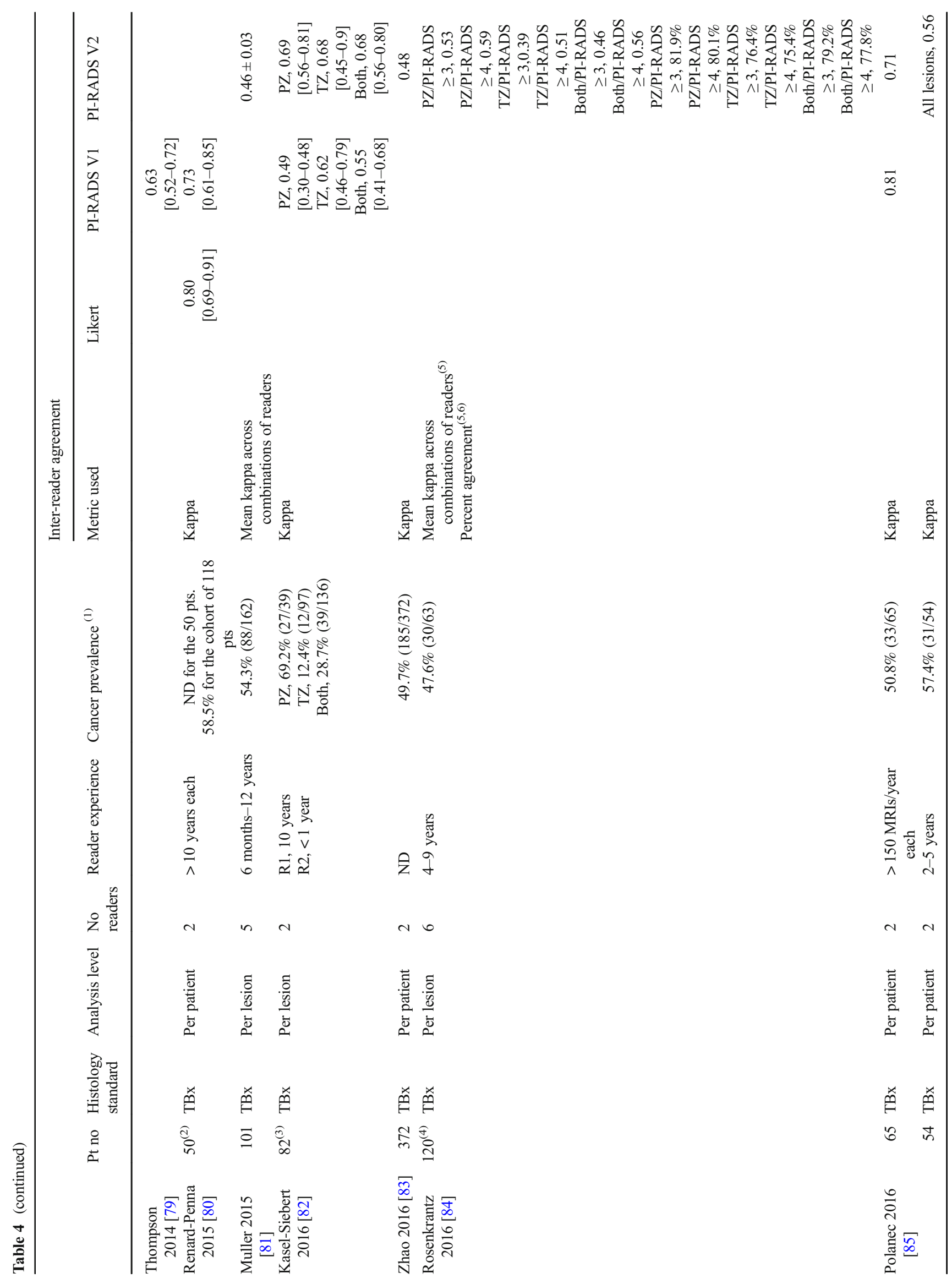




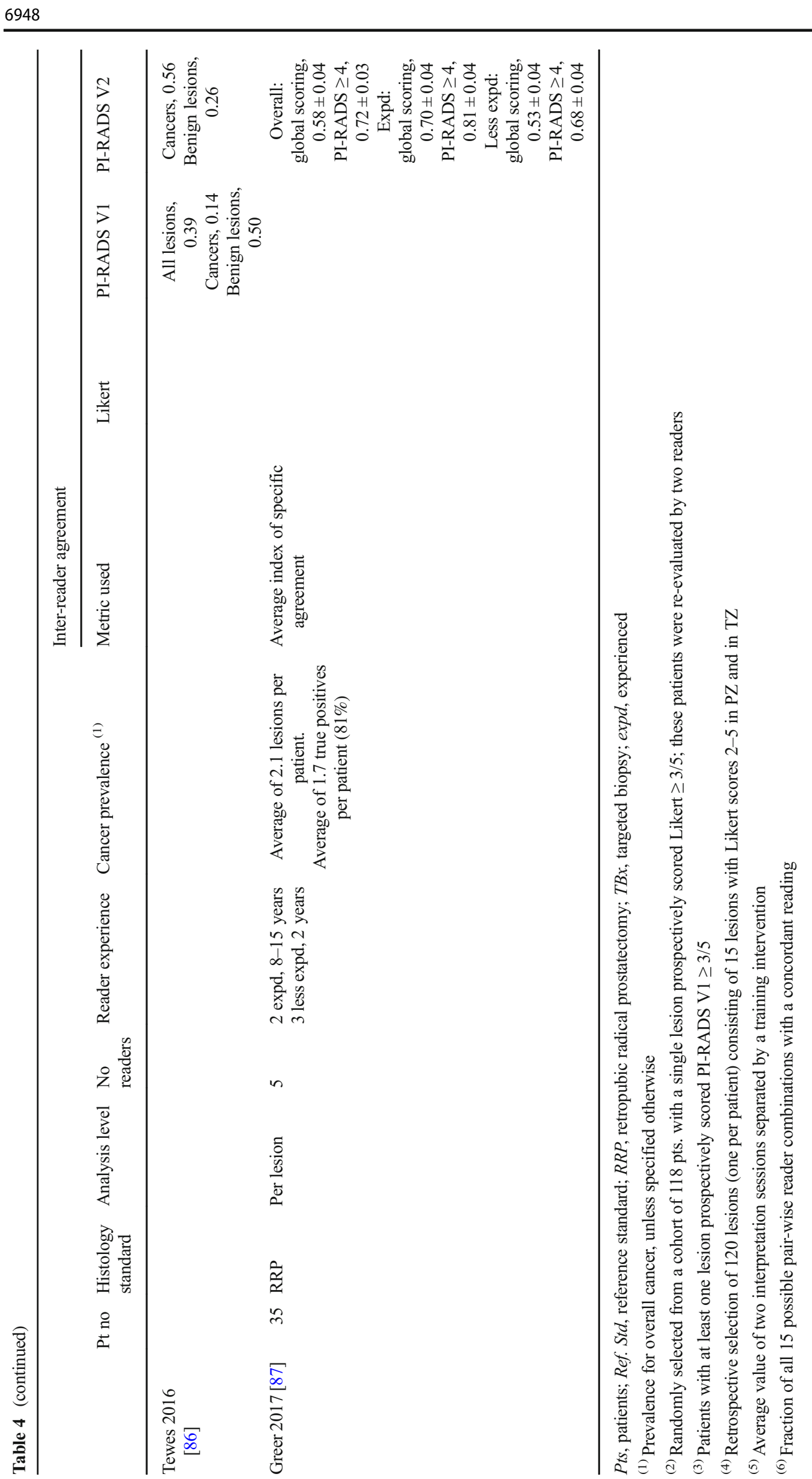

Eur Radiol (2019) 29:6940-6952 


\section{Inter-reader variability}

There are three difficulties with the widespread introduction of pre-biopsy mpMRI: the variable NPV of mpMRI, the variable accuracy of using mpMRI with TRUS to target suspicious lesions regardless of their location within the prostate gland, and inter-reader variability. The results from studies addressing the variability between 2013 and 2017 are summarised in Table 4.

The conclusions from the studies are succinctly captured by Hansen et al [88]: (1) mpMRI exams are more often called negative in subspecialist reads ( $41 \%$ vs $20 \%$ ); (2) second readings of prostate mpMRI by subspecialist uroradiologists significantly improve NPV and PPV; (3) reporter experience may reduce overcalling and avoid over targeting of lesions; and (4) greater education and training of radiologists in prostate mpMRI interpretation are advised. Many European countries are, in collaboration with ESUR and EAU, working to address this training need.

\section{Conclusion}

Current national guidelines in Europe highlight the worth of mpMRI in the management of men with suspected PCa. The case for using mpMRI to help in selecting which men with suspected PCa should have a biopsyand which need not-and to then select the regions of the prostate to biopsy (and which regions can be ignored) is compelling. The evidence base, including level 1 studies, is overpowering as are the arguments for patient benefit, avoiding either biopsy or overdiagnosis of clinically insignificant cancer.

Table 5 Suggestions for the use of mpMRI as a triage test in those with suspected prostate cancer

1. mpMRI should be the first investigation in the workup of men with suspected prostate cancer (Fig. 1)

2. PI-RADS assessment categories 1 and 2 have a high predictive value in excluding significant disease, and systematic biopsy may be postponed, especially in men with low-risk of disease following additional risk stratification (see 7 below)

3. PI-RADS assessment category lesions 4 and 5 should be targeted

4. PI-RADS assessment category lesion 3 may be targeted and systematic biopsied depending on risk stratification

5. Targeted biopsy (cognitive, MRI/US fusion, or 'in-bore') should be available for biopsy of focal lesions

6. Systematic biopsies in addition to targeted biopsy should be used judiciously rather than as a default, for example in cases being considered for focal therapy or nerve-sparing surgery

7. Where clinical risk parameters including age, family history, DRE findings, PSA velocity, and PSA density are of concern, SBx should be considered even in the setting of a negative mpMRI
Patients contemplating a biopsy are becoming aware that imaging by means of mpMRI may permit avoidance of biopsy in some cases and targeting in others. These patients will be understandably anxious to avoid the risks of biopsy, or at least minimise the risks by having fewer biopsy samples. Going away from 'default' SBx to premeditated TBx judiciously and selectively complimented by SBx using a two-step risk evaluation offers the best compromise to reduce biopsy rates and reduce overdiagnosis of cisPCa while minimising the chances of missing csPCa. The evidence to expect to avoid SBx altogether even in the era of pre-biopsy mpMRI is weak [54].

Our summary suggestions are presented in Table 5.

Funding The authors state that this work has not received any funding.

\section{Compliance with ethical standards}

Guarantor The scientific guarantor of this publication is Jonathan Richenberg, MA BM Bch MRCP FRCR Hon Sen Lect BSMS, on behalf of ESUR Prostate Working Group.

Conflict of interest The authors of this manuscript declare no relationships with any companies, whose products or services may be related to the subject matter of the article.

Statistics and biometry No complex statistical methods were necessary for this paper.

Informed consent No human subjects involved.

Ethical approval Institutional Review Board approval was not required because this is a review article of evidence published.

\section{Methodology}

- Literature review and evidence analysis.

- Multicentre study - ESUR Working Group, Prostate Cancer with representatives from the UK, France, Denmark, Italy, and Netherlands

Open Access This article is distributed under the terms of the Creative Commons Attribution 4.0 International License (http:// creativecommons.org/licenses/by/4.0/), which permits unrestricted use, distribution, and reproduction in any medium, provided you give appropriate credit to the original author(s) and the source, provide a link to the Creative Commons license, and indicate if changes were made.

\section{References}

1. Barentsz JO, Richenberg J, Clements R et al (2012) ESUR prostate MR guidelines 2012. Eur Radiol. https://doi.org/10.1007/s00330011-2377-y

2. Mottet N, van den Bergh RCN, Briers E et al (2018) Guidelines on prostate cancer. https://uroweb.org/guideline/prostate-cancer/? type $=$ archive

3. D'Amico AV, Whittington R, Malkowicz SB et al (1998) Biochemical outcome after radical prostatectomy, external beam 
radiation therapy, or interstitial radiation therapy for clinically localized prostate cancer. JAMA 280:969-974

4. D'Amico AV, Renshaw AA, Cote K et al (2004) Impact of the percentage of positive prostate cores on prostate cancer-specific mortality for patients with low or favorable intermediate-risk disease. J Clin Oncol 22:3726-3732

5. Linson PW, Lee AK, Doytchinova Tet al (2002) Percentage of core lengths involved with prostate cancer: does it add to the percentage of positive prostate biopsies in predicting postoperative prostatespecific antigen outcome for men with intermediate-risk prostate cancer. Urology 59:704-708

6. Freedland SJ, Aronson WJ, Csathy GS et al (2003) Comparison of percentage of total prostate needle biopsy tissue with cancer to percentage of cores with cancer for predicting PSA recurrence after radical prostatectomy: results from the SEARCH database. Urology 61:742-747

7. Hu JC, Chang E, Natarajan S et al (2014) Targeted prostate biopsy in select men for active surveillance: do the Epstein criteria still apply? J Urol 192:385-390

8. Epstein JI (2010) An update of the Gleason grading system. J Urol 183:433-440

9. Fütterer JJ, Briganti A, De Visschere P et al (2015) Can clinically significant prostate cancer be detected with multiparametric magnetic resonance imaging? A systematic review of the literature. Eur Urol 68:1045-1053

10. Epstein JI, Zelefsky MJ, Sjoberg DD et al (2016) A contemporary prostate cancer grading system: a validated alternative to the Gleason score. Eur Urol 69:428-435

11. Gulati R, Cheng HH, Lange PH, Nelson PS, Etzioni R (2017) Screening men at increased risk for prostate Cancer diagnosis: model estimates of benefits and harms. Cancer Epidemiol Biomarkers Prev 26:222-227

12. Bratan F, Niaf E, Melodelima $C$ et al (2013) Influence of imaging and histological factors on prostate cancer detection and localisation on multiparametric MRI: a prospective study. Eur Radiol 23:2019-2029

13. Selnaes KM, Heerschap A, Jensen LR et al (2012) Peripheral zone prostate cancer localization by multiparametric magnetic resonance at $3 \mathrm{~T}$ : unbiased cancer identification by matching to histopathology. Invest Radiol 47:624-633

14. Turkbey B, Mani H, Shah V et al (2011) Multiparametric 3T prostate magnetic resonance imaging to detect cancer: histopathological correlation using prostatectomy specimens processed in customized magnetic resonance imaging based molds. J Urol 186:1818-1824

15. Le JD, Tan N, Shkolyar E et al (2015) Multifocality and prostate cancer detection by multiparametric magnetic resonance imaging: correlation with whole-mount histopathology. Eur Urol 67:569-576

16. Hambrock T, Hoeks C, Hulsbergen-van de Kaa C et al (2012) Prospective assessment of prostate cancer aggressiveness using 3$\mathrm{T}$ diffusion-weighted magnetic resonance imaging-guided biopsies versus a systematic 10-core transrectal ultrasound prostate biopsy cohort. Eur Urol 61:177-184

17. Kobus T, Hambrock T, Hulsbergen-van de Kaa CA et al (2011) In vivo assessment of prostate cancer aggressiveness using magnetic resonance spectroscopic imaging at $3 \mathrm{~T}$ with an endorectal coil. Eur Urol 60:1074-1080

18. Wang L, Mazaheri Y, Zhang J, Ishill NM, Kuroiwa K, Hricak H (2008) Assessment of biologic aggressiveness of prostate cancer: correlation of MR signal intensity with Gleason grade after radical prostatectomy. Radiology 246:168-176

19. Bratan F, Melodelima C, Souchon R et al (2015) How accurate is multiparametric MR imaging in evaluation of prostate cancer volume. Radiology 275:144-154

20. Sun C, Chatterjee A, Yousuf A et al (2019) Comparison of T2weighted imaging, DWI, and dynamic contrast-enhanced MRI for calculation of prostate cancer index lesion volume: correlation with whole-mount pathology. AJR Am J Roentgenol 212:351-356
21. Weinreb JC, Barentsz JO, Choyke PL et al (2016) PI-RADS prostate imaging - reporting and data system: 2015, version 2. Eur Urol 69:16-40

22. Barentsz JO, Weinreb JC, Verma S et al (2016) Synopsis of the PIRADS v2 guidelines for multiparametric prostate magnetic resonance imaging and recommendations for use. Eur Urol 69:41-49

23. Woo S, Suh CH, Kim SY, Cho JY, Kim SH (2017) Diagnostic performance of prostate imaging reporting and data system version 2 for detection of prostate cancer: a systematic review and diagnostic meta-analysis. Eur Urol 72:177-188

24. Carroll PH, Mohler JL (2018) NCCN guidelines update: prostate cancer and prostate cancer early detection. J Nat Comp Canc Netw $16(5 \mathrm{~S}): 620-623$

25. NICE (2014) Prostate cancer: diagnosis and management. Available via https://www.nice.org.uk/guidance/cg175. Accessed on 7 March 2019

26. Rozet F, Hennequin C, Beauval JB et al (2018) French ccAFU guidelines - update 2018-2020: prostate cancer. Prog Urol 28: S79-S130

27. Donovan JL, Young GJ, Walsh EI et al (2018) A prospective cohort and extended comprehensive-cohort design provided insights about the generalizability of a pragmatic trial: the Protec $\mathrm{T}$ prostate cancer trial. J Clin Epidemiol 96:35-46

28. Mambourg F, Jonckheer P, Piérart J, Van Brabandt H (2012) A national clinical practice guideline on the management of localised prostate cancer. Belgian Health Care Knowledge Centre (KCE), KCE reports 194C. http://kce.fgov.be/sites/default/files/page_ documents/KCE_194C_prostate_cancer_0.pdf

29. Rozet F, Hennequin C, Beauval JB et al (2016) CCAFU French national guidelines 2016-2018 on prostate cancer. Prog Urol 27(Suppl 1):S95-S143

30. de Rooij M, Crienen S, Witjes JA, Barentsz JO, Rovers MM, Grutters JP (2014) Cost-effectiveness of magnetic resonance (MR) imaging and MR-guided targeted biopsy versus systematic transrectal ultrasound-guided biopsy in diagnosing prostate cancer: a modelling study from a health care perspective. Eur Urol 66:430-436

31. Faria R, Soares MO, Spackman E et al (2018) Optimising the diagnosis of prostate cancer in the era of multiparametric magnetic resonance imaging: a cost-effectiveness analysis based on the prostate MR imaging study (PROMIS). Eur Urol 73:23-30

32. Moldovan PC, Van den Broeck T, Sylvester R et al (2017) What is the negative predictive value of multiparametric magnetic resonance imaging in excluding prostate Cancer at biopsy? A systematic review and meta-analysis from the European Association of Urology Prostate cancer Guidelines Panel. Eur Urol 72:250-266

33. Ahmed HU, El-Shater Bosaily A, Brown LC et al (2017) Diagnostic accuracy of multi-parametric MRI and TRUS biopsy in prostate cancer (PROMIS): a paired validating confirmatory study. Lancet 389:815-822

34. Kasivisvanathan V, Rannikko AS, Borghi M et al (2018) MRItargeted or standard biopsy for prostate-cancer diagnosis diagnosis. N Engl J Med. https://doi.org/10.1056/NEJMoa1801993

35. van der Leest M, Cornel E, Israël B et al (2018) Head-to-head comparison of transrectal ultrasound-guided prostate biopsy versus multiparametric prostate resonance imaging with subsequent magnetic resonance-guided biopsy in biopsy-naive men with elevated prostate-specific antigen: a large prospective multicenter clinical study. Eur Urol. https://doi.org/10.1016/j.eururo.2018.11.023

36. Moore CM, Kasivisvanathan V, Eggener S et al (2013) Standards of reporting for MRI-targeted biopsy studies (START) of the prostate: recommendations from an International Working Group. Eur Urol 64:544-552

37. Venderink W, van Luijtelaar A, Bomers JG et al (2017) Results of targeted biopsy in men with magnetic resonance imaging lesions classified equivocal, likely or highly likely to be clinically 
significant prostate cancer. Eur Urol. https://doi.org/10.1016/j. eururo.2017.02.021

38. Panebianco V, Barchetti G, Simone G et al (2018) Negative multiparametric magnetic resonance imaging for prostate cancer: what's next? Eur Urol. https://doi.org/10.1016/j.eururo.2018.03.007

39. Baco E, Ukimura O, Rud E et al (2015) Magnetic resonance imaging-transectal ultrasound image-fusion biopsies accurately characterize the index tumor: correlation with step-sectioned radical prostatectomy specimens in 135 patients. Eur Urol 67:787-794

40. Delongchamps NB, Lefevre A, Bouazza N, Beuvon F, Legman P, Cornud F (2014) Detection of significant prostate cancer with magnetic resonance targeted biopsies-should transrectal ultrasoundmagnetic resonance imaging fusion guided biopsies alone be a standard of care? J Urol. https://doi.org/10.1016/j.juro.2014.11.002

41. Costa DN, Lotan Y, Rofsky NM et al (2016) Assessment of prospectively assigned Likert scores for targeted magnetic resonance imaging-transrectal ultrasound fusion biopsies in patients with suspected prostate cancer. J Urol 195:80-87

42. Habchi H, Bratan F, Paye A et al (2014) Value of prostate multiparametric magnetic resonance imaging for predicting biopsy results in first or repeat biopsy. Clin Radiol 69:e120-e128

43. Mozer P, Rouprêt M, Le Cossec C et al (2015) First round of targeted biopsies using magnetic resonance imaging/ ultrasonography fusion compared with conventional transrectal ultrasonography-guided biopsies for the diagnosis of localised prostate cancer. BJU Int 115:50-57

44. Cash H, Maxeiner A, Stephan C et al (2016) The detection of significant prostate cancer is correlated with the prostate imaging reporting and data system (PI-RADS) in MRI/transrectal ultrasound fusion biopsy. World J Urol 34:525-532

45. Schimmöller L, Quentin M, Arsov C et al (2014) MR-sequences for prostate cancer diagnostics: validation based on the PI-RADS scoring system and targeted MR-guided in-bore biopsy. Eur Radiol 24: 2582-2589

46. Mertan FV, Greer MD, Shih JH et al (2016) Prospective evaluation of the prostate imaging reporting and data system version 2 for prostate cancer detection. J Urol 196:690-696

47. Valerio M, Donaldson I, Emberton M et al (2015) Detection of clinically significant prostate cancer using magnetic resonance imaging-ultrasound fusion targeted biopsy: a systematic review. Eur Urol 68:8-19

48. Schoots IG, Roobol MJ, Nieboer D, Bangma CH, Steyerberg EW, Hunink MG (2015) Magnetic resonance imaging-targeted biopsy may enhance the diagnostic accuracy of significant prostate cancer detection compared to standard transrectal ultrasound-guided biopsy: a systematic review and meta-analysis. Eur Urol 68:438-450

49. Schouten MG, van der Leest M, Pokorny M et al (2017) Why and where do we miss significant prostate cancer with multi-parametric magnetic resonance imaging followed by magnetic resonanceguided and Transrectal ultrasound-guided biopsy in biopsy-naive men? Eur Urol 71:896-903

50. De Visschere PJ, Naesens L, Libbrecht L et al (2016) What kind of prostate cancers do we miss on multiparametric magnetic resonance imaging? Eur Radiol 26:1098-1107

51. Haffner J, Lemaitre L, Puech P et al (2011) Role of magnetic resonance imaging before initial biopsy: comparison of magnetic resonance imaging-targeted and systematic biopsy for significant prostate cancer detection. BJU Int 108:E171-E178

52. Moore CM, Robertson NL, Arsanious N et al (2013) Image-guided prostate biopsy using magnetic resonance imaging-derived targets: a systematic review. Eur Urol 63:125-140

53. Siddiqui MM, Rais-Bahrami S, Turkbey B et al (2015) Comparison of MR/ultrasound fusion-guided biopsy with ultrasound-guided biopsy for the diagnosis of prostate cancer. JAMA 313:390-397

54. Rouvière O, Puech P, Renard-Penna R et al (2019) Use of prostate systematic and targeted biopsy on the basis of multiparametric MRI in biopsy-naive patients (MRI-FIRST): a prospective, multicentre, paired diagnostic study. Lancet Oncol 20:100-109

55. Patel N, Cricco-Lizza E, Kasabwala K et al (2018) The role of systematic and targeted biopsies in light of overlap on magnetic resonance imaging ultrasound fusion biopsy. Eur Urol Oncol 1: 263-267

56. Radtke JP, Wiesenfarth M, Kesch C et al (2017) Combined clinical parameters and multiparametric magnetic resonance imaging for advanced risk modeling of prostate cancer-patient-tailored risk stratification can reduce unnecessary biopsies. Eur Urol 72:888-896

57. Distler FA, Radtke JP, Bonekamp D et al (2017) The value of PSA density in combination with PI-RADS for the accuracy of prostate cancer prediction. J Urol 198:575-582

58. Mehralivand S, Shih JH, Rais-Bahrami S et al (2018) A magnetic resonance imaging-based prediction model for prostate biopsy risk stratification. JAMA Oncol 4:678-685

59. Schoots IG (2018) MRI in early prostate cancer detection: how to manage indeterminate or equivocal PI-RADS 3 lesions? Transl Androl Urol 7:70-82

60. Felker ER, Raman SS, Margolis DJ et al (2017) Risk stratification among men with prostate imaging reporting and data system version 2 category 3 transition zone lesions: is biopsy always necessary? AJR Am J Roentgenol 209:1272-1277

61. Niu XK, Li J, Das SK, Xiong Y, Yang CB, Peng T (2017) Developing a nomogram based on multiparametric magnetic resonance imaging for forecasting high-grade prostate cancer to reduce unnecessary biopsies within the prostate-specific antigen gray zone. BMC Med Imaging 17:11

62. Shukla-Dave A, Hricak H, Akin O et al (2012) Preoperative nomograms incorporating magnetic resonance imaging and spectroscopy for prediction of insignificant prostate cancer. BJU Int 109:13151322

63. Vilanova JC, Barceló-Vidal C, Comet J et al (2011) Usefulness of prebiopsy multifunctional and morphologic MRI combined with free-to-total prostate-specific antigen ratio in the detection of prostate cancer. AJR Am J Roentgenol 196:W715-W722

64. Hansen NL, Barrett T, Koo B et al (2017) The influence of prostatespecific antigen density on positive and negative predictive values of multiparametric magnetic resonance imaging to detect Gleason score $7-10$ prostate cancer in a repeat biopsy setting. BJU Int 119: 724-730

65. Washino S, Okochi T, Saito K et al (2017) Combination of prostate imaging reporting and data system (PI-RADS) score and prostatespecific antigen (PSA) density predicts biopsy outcome in prostate biopsy naive patients. BJU Int 119:225-233

66. Liddell H, Jyoti R, Haxhimolla HZ (2015) mp-MRI prostate characterised PIRADS 3 lesions are associated with a low risk of clinically significant prostate cancer - a retrospective review of 92 biopsied PIRADS 3 lesions. Curr Urol 8:96-100

67. Hansen NL, Barrett T, Kesch C et al (2018) Multicentre evaluation of magnetic resonance imaging supported transperineal prostate biopsy in biopsy-naive men with suspicion of prostate cancer. BJU Int 122:40-49

68. Abd-Alazeez M, Kirkham A, Ahmed HU et al (2014) Performance of multiparametric MRI in men at risk of prostate cancer before the first biopsy: a paired validating cohort study using template prostate mapping biopsies as the reference standard. Prostate Cancer Prostatic Dis 17:40-46

69. Arsov C, Quentin M, Rabenalt R, Antoch G, Albers P, Blondin D (2012) Repeat transrectal ultrasound biopsies with additional targeted cores according to results of functional prostate MRI detects high-risk prostate cancer in patients with previous negative biopsy and increased PSA - a pilot study. Anticancer Res 32: 1087-1092 
70. de Rooij M, Hamoen EH, Fütterer JJ, Barentsz JO, Rovers MM (2014) Accuracy of multiparametric MRI for prostate cancer detection: a meta-analysis. AJR Am J Roentgenol 202:343-351

71. Grey AD, Chana MS, Popert R, Wolfe K, Liyanage SH, Acher PL (2014) Diagnostic accuracy of magnetic resonance imaging (MRI) prostate imaging reporting and data system (PI-RADS) scoring in a transperineal prostate biopsy setting. BJU Int. https://doi.org/10. 1111/bju. 12862

72. Pokorny MR, de Rooij M, Duncan E et al (2014) Prospective study of diagnostic accuracy comparing prostate cancer detection by transrectal ultrasound-guided biopsy versus magnetic resonance (MR) imaging with subsequent MR-guided biopsy in men without previous prostate biopsies. Eur Urol 66:22-29

73. Portalez D, Mozer P, Cornud F et al (2012) Validation of the European Society of Urogenital Radiology scoring system for prostate cancer diagnosis on multiparametric magnetic resonance imaging in a cohort of repeat biopsy patients. Eur Urol 62:986-996

74. Rosenkrantz AB, Mussi TC, Borofsky MS, Scionti SS, Grasso M, Taneja SS (2012) 3.0 T multiparametric prostate MRI using pelvic phased-array coil: utility for tumor detection prior to biopsy. Urol Oncol. https://doi.org/10.1016/j.urolonc.2012.02.018

75. Vargas HA, Akin O, Afaq A et al (2012) Magnetic resonance imaging for predicting prostate biopsy findings in patients considered for active surveillance of clinically low risk prostate cancer. J Urol 188:1732-1738

76. Rosenkrantz AB, Kim S, Lim RP et al (2013) Prostate cancer localization using multiparametric MR imaging: comparison of prostate imaging reporting and data system (PI-RADS) and Likert scales. Radiology 269:482-492

77. Rosenkrantz AB, Lim RP, Haghighi M, Somberg MB, Babb JS, Taneja SS (2013) Comparison of interreader reproducibility of the prostate imaging reporting and data system and likert scales for evaluation of multiparametric prostate MRI. AJR Am J Roentgenol 201:W612-W618

78. Vaché T, Bratan F, Mège-Lechevallier F, Roche S, Rabilloud M, Rouvière O (2014) Characterization of prostate lesions as benign or malignant at multiparametric MR imaging: comparison of three scoring systems in patients treated with radical prostatectomy. Radiology 272:446-455

79. Thompson JE, Moses D, Shnier R et al (2014) Multiparametric magnetic resonance imaging guided diagnostic biopsy detects sig- nificant prostate cancer and could reduce unnecessary biopsies and over detection: a prospective study. J Urol 192:67-74

80. Renard-Penna R, Mozer P, Cornud F et al (2015) Prostate imaging reporting and data system and Likert scoring system: multiparametric MR imaging validation study to screen patients for initial biopsy. Radiology 275:458-468

81. Muller BG, Shih JH, Sankineni S et al (2015) Prostate cancer: interobserver agreement and accuracy with the revised prostate imaging reporting and data system at multiparametric MR imaging. Radiology 277:741-750

82. Kasel-Seibert M, Lehmann T, Aschenbach R et al (2016) Assessment of PI-RADS v2 for the detection of prostate cancer. Eur J Radiol 85:726-731

83. Zhao C, Gao G, Fang D et al (2016) The efficiency of multiparametric magnetic resonance imaging (mpMRI) using PIRADS version 2 in the diagnosis of clinically significant prostate cancer. Clin Imaging 40:885-888

84. Rosenkrantz AB, Ginocchio LA, Cornfeld D et al (2016) Interobserver reproducibility of the PI-RADS version 2 lexicon: a multicenter study of six experienced prostate radiologists. Radiology 280:793-804

85. Polanec S, Helbich TH, Bickel H et al (2016) Head-to-head comparison of PI-RADS v2 and PI-RADS v1. Eur J Radiol 85:11251131

86. Tewes S, Mokov N, Hartung D et al (2016) Standardized reporting of prostate MRI: comparison of the prostate imaging reporting and data system (PI-RADS) version 1 and version 2. PLoS One 11: $\mathrm{e} 0162879$

87. Greer MD, Brown AM, Shih JH et al (2017) Accuracy and agreement of PIRADSv2 for prostate cancer mpMRI: a multireader study. J Magn Reson Imaging 45:579-585

88. Hansen NL, Koo BC, Gallagher FA et al (2017) Comparison of initial and tertiary centre second opinion reads of multiparametric magnetic resonance imaging of the prostate prior to repeat biopsy. Eur Radiol 27:2259-2266

Publisher's note Springer Nature remains neutral with regard to jurisdictional claims in published maps and institutional affiliations. 